\title{
Multiple-scale analysis of oscillatory thermocapillary convection of high Prandtl number fluids in a rectangular cavity
}

\author{
Chun-Liang Lai * \\ Department of Mechanical Engineering, National Taiwan University, Taipei 106, Taiwan, ROC \\ Received 30 December 2002; received in revised form 25 July 2003
}

\begin{abstract}
A lot of research work has been directed toward the study of oscillatory thermocapillary convection for the past about 20 years. However, the real mechanism for the onset of oscillatory thermocapillary convection is still not fully understood. The reasons are as follows. If, to most people, the Marangoni number, $M a$, is the only parameter to describe the onset of oscillatory thermocapillary convection, why it cannot correlate the ground-based and microgravity experimental data properly? If the "surface-deformation number" or the so-called " $S$-parameter" is the parameter which can correlate the experimental data properly, why it cannot be derived without conjectures?

To resolve this deficiency, multiple-scale analysis is applied to determine, among others, the characteristic length, time, and velocity scales for an unsteady, two-dimensional thermocapillary convection with a deformable free surface in a rectangular cavity. For flow situations with $A^{2} M_{a, 0} \gg \mathrm{O}(1), A^{2} R_{\sigma, 0} \gg \mathrm{O}(1)$, and $\operatorname{Pr}>\mathrm{O}(1)$, the ratio of the free surface-variation time scale to the convective time scale of the main surface flow turns out to be the so-called " $S$ parameter". The $S$-parameter thus implies physically a delayed cooling effect of the return flow on the surface flow. Therefore, the most important contribution of the present study is to provide a theoretical basis for the derivation of the $S$-parameter and, hopefully, guidance for the study of oscillatory thermocapillary convection.
\end{abstract}

(C) 2003 Elsevier Ltd. All rights reserved.

Keywords: Multiple-scale analysis; Thermocapillary convection; Surface deformation; $S$-parameter

\section{Introduction}

The surface tension of an interface between two immiscible liquids or of a free surface between a liquid and a passive gas depends generally on temperature, composition, and electrical potential. In many cases, it is a monotonically decreasing function of temperature. Therefore, any temperature gradient along the interface or free surface will, without the existence of contamination, generate a surface-tension gradient and hence a bulk fluid motion through the viscous diffusion. Such a fluid motion is called thermocapillary convection. Thermocapillary convection can be found in many ap-

\footnotetext{
Tel.: +886-2-2363-4820; fax: +886-2-2363-1755.

E-mail address: cllai@ccms.ntu.edu.tw (C.-L. Lai).
}

plications as an important or even a dominant effect, especially in small scale systems or microgravity environments [1]. Examples are migration of a droplet or a bubble in a nonuniform temperature field [2-4], flame spreading over pools of liquid fuel [5], welding [6], crystal growth from the melt [7,8], etc. Studies of flows induced by surface-tension gradients along an interface or free surface that were made up to the early 1970s were summarized by Ostrach [9].

Because of its importance from both the academic and application points of view, serious attention has been given to the studies of thermocapillary convections since the late 1970s. Most of the studies were related to the crystal-growth flows, especially on the transition from steady to oscillatory thermocapillary flows, and were studied experimentally [10-14], analytically [15-20], and numerically [21-29]. 


$\begin{array}{llll}\text { Nomenclature } & & \\ & & & \\ A & \text { aspect ratio } & \Delta T_{0} & \text { reference temperature difference } \\ C a & \text { capillary number } & t & \text { dimensionless time } \\ D & \text { original depth of liquid layer } & (U, V) & \text { velocity vector } \\ H & \text { instant height of liquid layer } & \left(U_{0}, V_{0}\right) & \text { reference velocity vector } \\ h & \text { dimensionless height of liquid layer } & \left(U_{\mathrm{r}}, V_{\mathrm{r}}\right) & \text { velocity vector of return flow } \\ L & \text { length of rectangular cavity } & U_{\mathrm{S}} & \text { surface velocity } \\ M a & \text { Marangoni number } & (u, v) & \text { dimensionless velocity vector } \\ M_{a, 0} & \text { Marangoni number conventionally adopted } & (X, Y) & \text { coordinate system } \\ M_{a, \mathrm{~S}} & \text { Marangoni number based on actual surface } & (x, y) & \text { dimensionless coordinate system } \\ & \text { velocity } & \alpha & \text { thermal diffusivity } \\ P & \text { pressure } & \Delta & \text { small length scale near side walls } \\ P_{0} & \text { reference pressure } & \delta & \text { boundary layer thickness } \\ P_{\infty} & \text { ambient pressure } & \mu & \text { dynamic viscosity } \\ p & \text { dimensionless pressure } & v & \text { kinematic viscosity } \\ P r & \text { Prandtl number } & \sigma & \text { dimensionless surface tension } \\ R_{\sigma} & \text { surface-tension Reynolds number } & \hat{\sigma} & \text { surface tension } \\ R_{\sigma, 0} & \text { surface-tension Reynolds number conven- } & \hat{\sigma}_{0} & \text { reference surface tension } \\ & \text { tionally adopted } & \hat{\sigma}_{\mathrm{T}} & \text { surface-tension derivative with respect to } \\ R_{\sigma, \mathrm{S}} & \text { surface-tension Reynolds number based on } & & \text { temperature } \\ & \text { actual surface velocity } & \tau & \text { time } \\ T & \text { temperature } & \tau_{0} & \text { reference time } \\ T_{\mathrm{H}} & \text { higher temperature } & \theta & \text { dimensionless temperature } \\ T_{\mathrm{L}} & \text { lower temperature } & & \\ & & & \end{array}$

However, the physical mechanism for the onset of oscillatory thermocapillary flows are still not fully understood and convincing due to the complexity of the problem itself and, probably, the inappropriate approach of the analysis. The reason for this argument is that if the Marangoni number, $M a$, or the surface-tension Reynolds number, $R_{\sigma}$, is the only parameter to describe the onset of oscillatory thermocapillary convection, why it cannot correlate the ground-based and microgravity experimental data properly? If the "surface-deformation number" or the so-called " $S$-number" is the parameter which can correlate the experimental data properly [30-32], why it cannot be derived without conjectures? The complexities and difficulties of the unsteady thermocapillary convection mainly come from the nonlinearity of the problem and the coupling between the velocity and temperature fields through the governing equations and boundary conditions imposed on the interface or free surface. As a result, the temperature distributions and the induced flow structures may be quite different from one parameter regime to another. Surface deformation makes the problem even more formidable. Conventional treatments may therefore possess deficiency in exploring the essential physics for such a complicated problem. Hence, in order to help formulating and solving the problem properly, phenomenological study based on the multiple-scale analysis seems to be an appropriate first-step approach.
Moreover, thermocapillary convection in a confined container is multiple-scaled in nature. The energy equation determines the length scales of the temperature distribution while the momentum equation determines the length scales of the velocity field. Surface deformation may generate another length scale which is usually much smaller than the length scales of the temperature and velocity fields. The time scales for the temperature, velocity, and surface variations may be different or the same depending on the transport mechanism. Before the detailed derivation of the various scales of the problem is given, a global picture about the multiple-scaled nature of the thermocapillary convection in a rectangular cavity will be depicted first in this section.

Since the surface temperature distribution is the driving mechanism for thermocapillary convection, the length scales of the temperature field will be discussed first. As shown in Fig. 1, depending on the heat-transfer mechanism, there may exist three different types of surface temperature distribution. When conduction is dominant or about the same order as convection, the surface temperature varies gradually from the high temperature, $T_{\mathrm{H}}$, at the left wall to the low temperature, $T_{\mathrm{L}}$, at the right wall. The geometric length scales, $(L, D)$, will be the proper ones for the temperature field. As convection becomes more and more important, a rapid change of the surface temperature distribution will appear first near the low temperature or cold wall at right. 


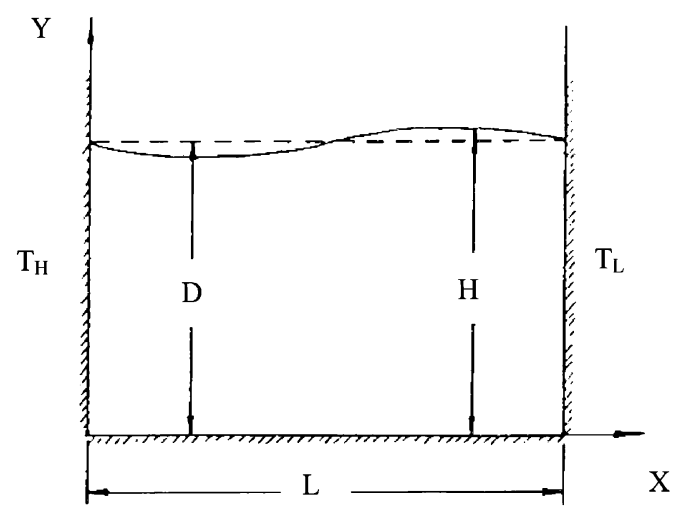

(a)

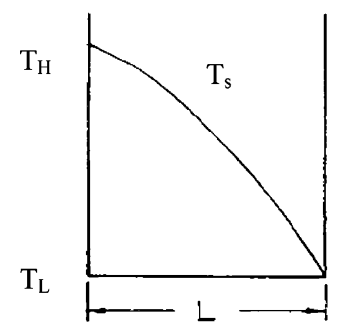

(b)

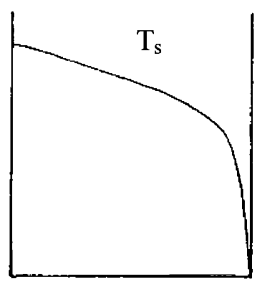

(c)

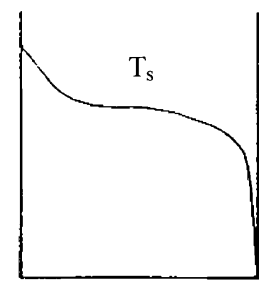

(d)

Fig. 1. (a) The geometrical configuration and coordinate system, (b) uniform surface temperature variation, (c) rapid surface temperature variation near the cold wall and (d) rapid surface temperature variation near both the hot and cold walls.

It is obvious that another length scale will be necessary to describe properly the temperature variation in that region. Moreover, since the surface temperature distribution is the driving mechanism for the flow, the flow behavior in the small region near the cold wall will be different from that in the other portion of the cavity. As convection becomes much stronger than conduction, the surface temperature distribution may change rapidly near both the hot (high temperature) and cold walls. In such a case, three length scales may exist for the temperature field; one is for the region near the hot wall, another for the region close to the cold wall, and the third for the central portion of the cavity where the surface temperature variation becomes very mild.

Similar to the temperature field, the length scales of the velocity field are determined by the mechanism of momentum transport. For any of the three possibilities of the surface temperature distribution, if the flow generated by the thermocapillarity is inertia dominant there will exist a velocity boundary layer near the free surface, which needs another length scale to describe properly the flow behavior therein. If the flow is of viscous type, the geometric depth of the liquid, $D$, will be one of the length scale of the velocity field. Of course, other length scales may exist near either the cold wall or the hot wall in accordance with the surface temperature distribution.
In addition to the length scales of the temperature and velocity fields, the surface deformation will generate another length scale which must be properly determined from the normal-force-balance boundary condition at the free surface.

In the present study, the various space and time scales for the temperature and velocity fields and the surface variation as described above will be derived through scaling analysis by proper balances between physical terms in either the governing equations or the boundary conditions. The scaling analysis was first applied to the thermocapillary convection by Ostrach [33]. However, the possible changes of the surface temperature distribution and the flow structure for different flow regimes were not considered. Various length and time scales for a half-zone configuration with a deformable free surface were first investigated by Lai [30]. Parameters for predicting the onset of oscillatory thermocapillary flows were derived thereby. More detailed analysis for the flow structures and temperature distributions of steady thermocapillary flows in a square cavity with a nondeformable free surface was pursued later by $\mathrm{Hu}$ [34]. Although various scaling groups for different flow regimes were obtained by Lai [30] and $\mathrm{Hu}$ [34], several conjectures on the physical arguments were applied during the scaling analysis, which makes the 
derivation of the dimensionless parameters not fully convincing. Kamotani and Ostrach [31] rederived the so-called $S$-parameter. However, the final dimensionless parameter obtained was modified by a small power of $M a$ and the aspect-ratio effect was not considered in the analysis. The present study is therefore aimed at resolving these deficiencies. Various scales for the temperature field, velocity field, and surface deformation and the relevant dimensionless parameters are derived properly. A clearer picture about the physical mechanism for the onset of oscillatory thermocapillary convection is thus provided.

\section{Mathematical formulation}

\subsection{Governing equations and boundary conditions}

The system to be considered is a two-dimensional rectangular cavity of length $L$ filled with an incompressible liquid of depth $D$. The schematic diagram of the physical model and the associated coordinate system $(X, Y)$ are shown in Fig. 1. The left wall of the cavity is maintained at a higher temperature $T_{\mathrm{H}}$ with the right one at a lower temperature $T_{\mathrm{L}}$. A thermally insulated boundary condition is applied at both the bottom wall and the free surface. As time proceeds, thermal energy is transferred from the left wall to the right one, resulting in a nonuniform temperature distribution along the free surface. Bulk flow is then generated through the thermocapillary effect.

The following assumptions are made to simplify the problem:

(1) The induced thermocapillary flows are two-dimensional and incompressible.

(2) Gravitational force and the induced natural convection are negligibly small compared to the thermocapillary effects.

(3) Free surface deformation is small.

(4) The free surface and bottom wall are thermally insulated.

(5) Viscous dissipation is neglected.

(6) The contact angle of the free surface at the end walls are $90^{\circ}$

Before the detailed derivation of the characteristic quantities is presented in next section, the nondimensionalization scheme applied conventionally will be given first in the following.

Let $(x, y),(u, v), t, p, \theta, h$, and $\sigma$ represent, respectively, the dimensionless forms of the coordinate system $(X, Y)$, the velocity vector $(U, V)$, time $\tau$, pressure $P$, temperature $T$, the free surface height $H$, and the surface tension $\hat{\sigma}$. The nondimensionalization scheme conventionally applied is as follows:

$$
\begin{aligned}
& x=\frac{X}{L}, \quad y=\frac{Y}{D}, \quad t=\frac{\tau}{\tau_{0}}, \quad u=\frac{U}{U_{0}}, \quad v=\frac{V}{V_{0}}, \\
& h=\frac{H}{D}, \quad p=\frac{P-P_{\infty}}{P_{0}}, \quad \theta=\frac{T-T_{\mathrm{L}}}{\Delta T_{0}}, \quad \sigma=\frac{\hat{\sigma}}{\hat{\sigma}_{0}} .
\end{aligned}
$$

In the above expressions, $U_{0}$ and $V_{0}$ represent, respectively, the characteristic velocities in the $X$ - and $Y$-directions; $P_{0}$, the reference pressure; $\Delta T_{0}=T_{\mathrm{H}}-T_{\mathrm{L}}$, the reference temperature difference; $\tau_{0}$, the reference time scale, and $\hat{\sigma}_{0}=\hat{\sigma}_{0}\left(\frac{T_{\mathrm{H}}+T_{\mathrm{L}}}{2}\right)$, the reference surface tension. $P_{\infty}$ is the ambient pressure. The reference quantities $U_{0}$, $V_{0}, P_{0}$, and $\tau_{0}$ are usually determined from a viscous flow consideration with conduction dominant. Thus, $U_{0}=$ $A\left|\hat{\sigma}_{\mathrm{T}}\right| \Delta T_{0} / \mu, V_{0}=A U_{0}, P_{0}=L \mu U_{0} / D^{2}$, and $\tau_{0}=D^{2} / \alpha$, with $A=D / L$ denoting the aspect ratio and $\hat{\sigma}_{\mathrm{T}}=\partial \hat{\sigma} / \partial T$, the surface-tension derivative with respect to temperature.

Under the assumptions made above, the dimensionless governing equations and boundary conditions for the flow system considered herein possess the following forms:

$\frac{\partial u}{\partial x}+\frac{\partial v}{\partial y}=0$

$\frac{1}{\operatorname{Pr}} \frac{\partial u}{\partial t}+A^{2} R_{\sigma, 0}\left(u \frac{\partial u}{\partial x}+v \frac{\partial u}{\partial y}\right)=-\frac{\partial p}{\partial x}+\left(A^{2} \frac{\partial^{2} u}{\partial x^{2}}+\frac{\partial^{2} u}{\partial y^{2}}\right)$

$\frac{1}{\operatorname{Pr}} \frac{\partial v}{\partial t}+A^{2} R_{\sigma, 0}\left(u \frac{\partial v}{\partial x}+v \frac{\partial v}{\partial y}\right)=-\frac{1}{A^{2}} \frac{\partial p}{\partial y}+\left(A^{2} \frac{\partial^{2} v}{\partial x^{2}}+\frac{\partial^{2} v}{\partial y^{2}}\right)$

$\frac{\partial \theta}{\partial t}+A^{2} M_{a, 0}\left(u \frac{\partial \theta}{\partial x}+v \frac{\partial \theta}{\partial y}\right)=A^{2} \frac{\partial^{2} \theta}{\partial x^{2}}+\frac{\partial^{2} \theta}{\partial y^{2}}$

$u=0, \quad v=0, \quad \theta=1 \quad$ at $x=0$

$u=0, \quad v=0, \quad \theta=0 \quad$ at $x=1$

$u=0, \quad v=0, \quad \frac{\partial \theta}{\partial y}=0 \quad$ at $y=0$

At the free surface: (with the assumption of small surface deformation) normal-force-balance boundary condition:

$\left(A^{-2} C a\right) p-2 C a \frac{\partial v}{\partial y} \approx-\sigma \frac{\partial^{2} h}{\partial x^{2}}$

tangential-force-balance boundary condition:

$\frac{\partial u}{\partial y} \approx-\frac{\partial \theta}{\partial x}$

thermally insulated boundary condition:

$\frac{\partial \theta}{\partial y} \approx 0$ 
kinematic boundary condition:

$\left(\frac{1}{A^{2} M_{a, 0}}\right) \frac{\partial h}{\partial t}=v-u \frac{\partial h}{\partial x}$

or

$\left(\frac{1}{A^{2} M_{a, 0}}\right) \frac{\partial h}{\partial t}=-\frac{\partial}{\partial x}\left[\int_{0}^{h} u \mathrm{~d} y\right]$

The dimensionless parameters in the above expressions are defined as follows:

$A \equiv \frac{D}{L}=$ Aspect ratio

$\operatorname{Pr} \equiv \frac{v}{\alpha}=$ Prandtl number

$R_{\sigma, 0} \equiv \frac{\left|\hat{\sigma}_{\mathrm{T}}\right| \Delta T_{0} D}{\mu \nu}$

$=$ surface-tension Reynolds number

$M_{a, 0} \equiv \frac{\left|\hat{\sigma}_{T}\right| \Delta T_{0} D}{\mu \alpha}=\operatorname{Pr} R_{\sigma, 0}=$ Marangoni number

$C a \equiv \frac{\left|\hat{\sigma}_{\mathrm{T}}\right| \Delta T_{0}}{\hat{\sigma}_{0}}=$ Capillary number

The subscript " 0 " in $R_{\sigma, 0}$ and $M_{a, 0}$ is intentionally included to indicate that both parameters are based on the conventional scaling analysis. They will be used as references in next section where multiple-scale analysis is performed.

\subsection{Classification of flow regimes}

From the dimensional analysis as shown in Section 2.1, the flow regimes can be classified according to the order of magnitudes of $A^{2} M_{a, 0}$ and $A^{2} R_{\sigma, 0}$. Therefore, four flow regimes are possible; they are (i) $A^{2} M_{a, 0} \leqslant \mathrm{O}(1)$ and $A^{2} R_{\sigma, 0} \leqslant \mathrm{O}(1)$, (ii) $A^{2} M_{a, 0} \leqslant \mathrm{O}(1)$ and $A^{2} R_{\sigma, 0} \gg \mathrm{O}(1)$, (iii) $A^{2} M_{a, 0} \gg \mathrm{O}(1)$ and $A^{2} R_{\sigma, 0} \leqslant \mathrm{O}(1)$, and (iv) $A^{2} M_{a, 0} \gg$ $\mathrm{O}(1)$ and $A^{2} R_{\sigma, 0} \gg \mathrm{O}(1)$.

For case (i) with $A^{2} M_{a, 0} \leqslant \mathrm{O}(1)$ and $A^{2} R_{\sigma, 0} \leqslant \mathrm{O}(1)$, conduction is dominant and the flow is of viscous type. The geometric lengths will be the proper length scales for both the temperature and velocity fields. For case (ii) with $A^{2} M_{a, 0} \leqslant \mathrm{O}(1)$ and $A^{2} R_{\sigma, 0} \gg \mathrm{O}(1)$, conduction is still the dominant mechanism for energy transport while the flow is now of boundary-layer type. The length scale for the velocity field in the $Y$-direction and near the free surface should be re-scaled with the velocity boundary layer thickness. For case (iii) and (iv) with $A^{2} M_{a, 0} \gg$ $\mathrm{O}(1)$, the geometric length scales become improper to the temperature field. All the length scales and other reference quantities have to be reconsidered based on appropriate physical reasoning. The analysis becomes much more complicated and has been completely done by Lai [35]. However, since the onsets of oscillatory thermocapillary convection were observed and measured both on ground and in space for the flow situation with $A^{2} M_{a, 0} \gg \mathrm{O}(1), A^{2} R_{\sigma, 0} \gg \mathrm{O}(1)$, and $P r>\mathrm{O}(1)$, Only the multiple-scale analysis for this flow situation will be given and discussed in the present study. People interested in the detailed derivation for all the four flow regimes can refer to the analysis done by Lai [35].

\section{Multiple-scale analysis}

As discussed in last section, only the flow situations with $A^{2} M_{a, 0} \gg \mathrm{O}(1), A^{2} R_{\sigma, 0} \gg \mathrm{O}(1)$, and $\operatorname{Pr}>\mathrm{O}(1)$ will be analyzed in this section.

As shown in Fig. 2, for liquids with $\operatorname{Pr}>\mathrm{O}(1)$, the velocity boundary layer along the hot wall, $\delta_{\mathrm{V}, \mathrm{h}}$, is thicker than the thermal boundary layer along the same wall, $\delta_{\mathrm{T}, \mathrm{h}}$. The cooler fluid outside the thermal boundary layer $\delta_{\mathrm{T}, \mathrm{h}}$ but within the velocity boundary layer $\delta_{\mathrm{V}, \mathrm{h}}$ is directed toward the free surface before it turns and flows downstream toward the cold wall. As a result, the return flow will provide a cooling effect on the surface flow and results in, consequently, a rapid descent of the surface temperature distribution near the hot wall. Therefore, for situations with $A^{2} R_{\sigma, 0} \gg \mathrm{O}(1)$ and $\operatorname{Pr}>\mathrm{O}(1)$ (then $A^{2} M_{a, 0} \gg \mathrm{O}(1)$ also), there will exist two small regions, with one close to the hot wall and the other close to the cold wall, wherein the surface temperature variation is large. Across the central portion of the cavity, the surface temperature variation is mild, resulting in the so-called "S-shaped" surface temperature distribution. With such an S-shaped surface temperature distribution, the driving force for the flow mainly comes from the surface-tension gradient in the small regions near the hot and cold walls. As it leaves the hot wall, the fluid is pulled by a strong surface-tension gradient and reaches a locally maximum velocity before it enters the central portion of the cavity, as shown in Fig. 2. Because the driving force is greatly reduced, the flow, when entering the central portion of the cavity, is decelerated by the retarding viscous stress. If the surface-tension gradient and the viscous shear stress are about the same order of magnitude, the surface flow might retain a nearly constant speed (the locally maximum velocity in the small region near the hot wall) crossing the central portion of the cavity. However, as the flow enters the small region near the cold wall, the fluid is suddenly accelerated and reaches a peak speed due to the strong surface-tension gradient therein. Therefore, although the flow can be divided into three sub-regions based on the S-shaped surface temperature distribution, there exist only two characteristic surface velocities across the whole cavity. The locally maximum velocity, $U_{\mathrm{S}}$, attained in the small region near the hot wall, i.e., $\Delta_{\mathrm{T}, \mathrm{h}}$, is the characteristic surface velocity for both the region $\Delta_{\mathrm{T}, \mathrm{h}}$ and the central portion of the cavity. The peak value of the surface velocity in the small region 


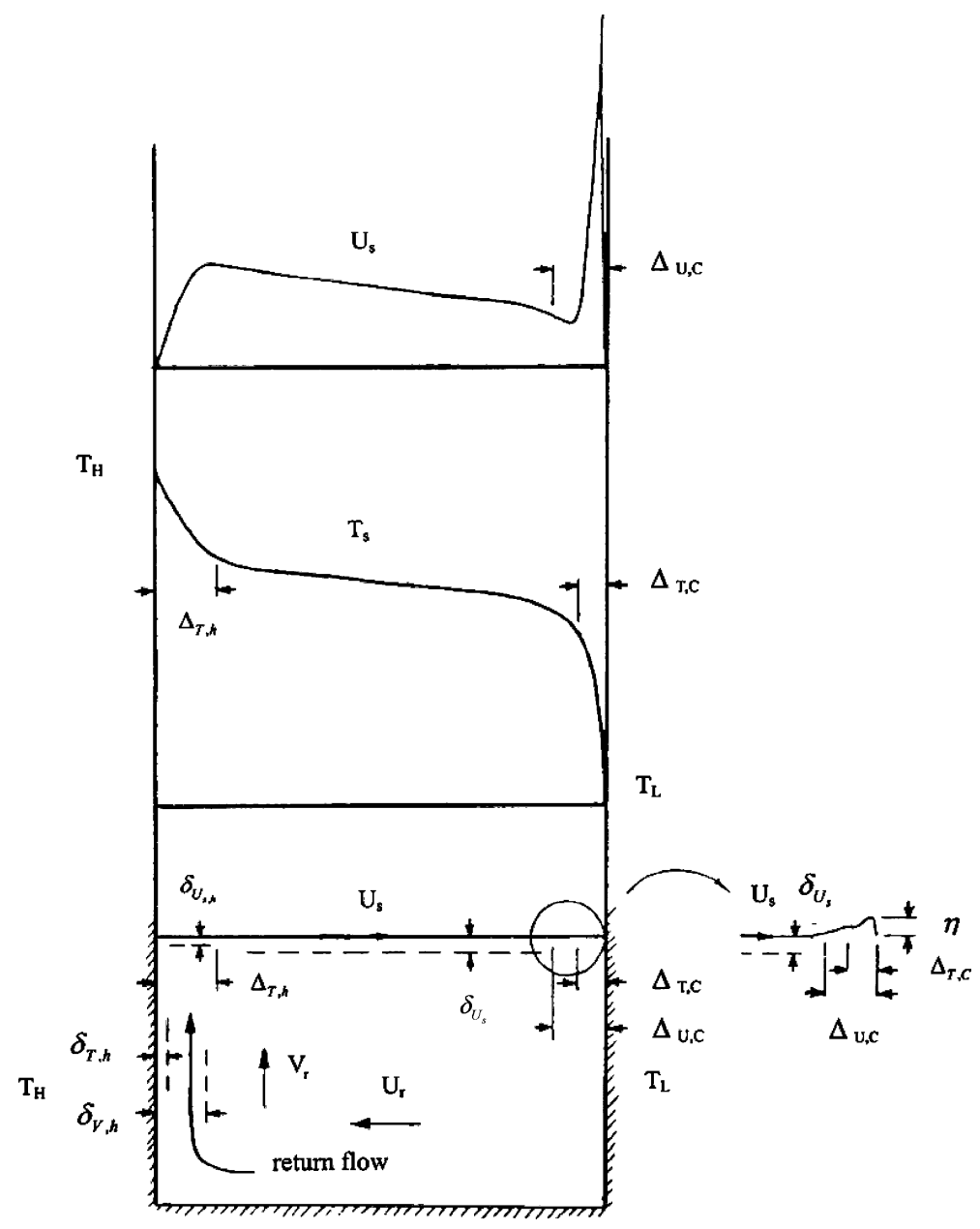

Fig. 2. The schematic diagrams of the surface temperature distribution, the surface velocity distribution, and other characteristic quantities for situations with $A^{2} M_{a, 0} \gg \mathrm{O}(1), A^{2} R_{\sigma, 0} \gg \mathrm{O}(1)$, and $\operatorname{Pr}>\mathrm{O}(1)$.

near the cold wall, i.e., $\Delta_{\mathrm{T}, \mathrm{C}}$, is another local characteristic velocity.

From the above discussion, it can be realized that the derivation of the characteristic quantities for the present situation is very complicated. Seven equations need to be solved simultaneously to determine the two most essential quantities, i.e., $\Delta_{\mathrm{T}, \mathrm{h}}$ and $U_{\mathrm{S}}$, among others. The seven relations are listed in the following:

$U_{\mathrm{S}} \frac{\Delta T_{0}}{\Delta_{\mathrm{T}, \mathrm{h}}} \sim \alpha \frac{\Delta T_{0}}{\delta_{\mathrm{T}_{\mathrm{S}} \mathrm{h}}^{2}}$

$V_{\mathrm{r}} \frac{\Delta T_{0}}{\delta_{\mathrm{T}_{\mathrm{S}}, \mathrm{h}}} \sim \alpha \frac{\Delta T_{0}}{\delta_{\mathrm{T}_{\mathrm{S}}, \mathrm{h}}^{2}}$

$U_{\mathrm{S}} \sim \frac{\delta_{U_{\mathrm{S}} \mathrm{h}}\left|\hat{\sigma}_{\mathrm{T}}\right| \Delta T_{0}}{\mu \Delta_{\mathrm{T}, \mathrm{h}}}$

$U_{\mathrm{r}} \sim \frac{\delta_{U_{\mathrm{S}}}}{D} U_{\mathrm{S}}$
$V_{\mathrm{r}} \sim A U_{\mathrm{r}}$

$\delta_{U_{\mathrm{S}}} \sim \frac{L}{\left(\frac{U_{\mathrm{S}} L}{v}\right)^{1 / 2}}$

$\delta_{U_{\mathrm{S}} \mathrm{h}} \sim \frac{\Delta_{\mathrm{T}, \mathrm{h}}}{\left(\frac{U_{\mathrm{S}} \Delta_{\mathrm{T}, \mathrm{h}}}{v}\right)^{1 / 2}}$

The physical meanings of the above relations are described as follows:

Eq. (18) is the balance between the convection in the $X$-direction and the conduction in the $Y$-direction near the free surface in $\Delta_{\mathrm{T}, \mathrm{h}}$.

Eq. (19) is the balance between the convection and conduction both in the $Y$-direction near the free surface in $\Delta_{\mathrm{T}, \mathrm{h}}$. The determination of $\delta_{\mathrm{T}_{\mathrm{S}} \mathrm{h}}$ is also an indication of the cooling effect of the return flow on the surface flow.

Eq. (20) represents the tangential-force-balance boundary condition at the free surface in $\Delta_{\mathrm{T}, \mathrm{h}}$. Eq. (21) 
is a relation of mass conservation between the surface flow and the return flow. Eq. (22) is from the continuity equation of the return flow. Eq. (23) represents the velocity boundary layer thickness along the free surface across the cavity. Eq. (24) is an estimation of the velocity boundary layer thickness along the free surface in $\Delta_{\mathrm{T}, \mathrm{h}}$.

There are seven unknowns, i.e., $\Delta_{\mathrm{T}, \mathrm{h}}, \delta_{\mathrm{T}_{\mathrm{S}} \mathrm{h}}, \delta_{U_{\mathrm{S}}}, \delta_{U_{\mathrm{S}}, \mathrm{h}}$, $U_{\mathrm{S}}, U_{\mathrm{r}}$, and $V_{\mathrm{r}}$, involved in Eqs. (18)-(24). Their physical meanings are shown in Fig. 2 and are described in the following:

$\Delta_{\mathrm{T}, \mathrm{h}} \quad \begin{aligned} & \text { The length scale of the surface temperature } \\ & \text { variation near the hot wall }\end{aligned}$
$\delta_{\mathrm{T}_{\mathrm{S}}, \mathrm{h}} \quad \begin{aligned} & \text { The thermal boundary layer thickness along } \\ & \text { the free surface in } \Delta_{\mathrm{T}, \mathrm{h}}\end{aligned}$
$\delta_{U_{\mathrm{S}}} \quad \begin{aligned} & \text { The velocity boundary layer thickness along } \\ & \text { the free surface across the cavity }\end{aligned}$
$\delta_{U_{\mathrm{S}}, \mathrm{h}} \quad \begin{aligned} & \text { The velocity boundary layer thickness along } \\ & \text { the free surface in } \Delta_{\mathrm{T}, \mathrm{h}}\end{aligned}$
$U_{\mathrm{S}} \quad \begin{aligned} & \text { The characteristic surface velocity for both the } \\ & \text { region } \Delta_{\mathrm{T}, \mathrm{h}} \text { and the central portion of the }\end{aligned}$
cavity
$U_{\mathrm{r}} \quad \begin{aligned} & \text { The characteristic velocity in the } X \text {-direction of } \\ & \text { the return flow }\end{aligned}$
$V_{\mathrm{r}} \quad \begin{aligned} & \text { The characteristic velocity in the } Y \text {-direction of } \\ & \text { the return flow }\end{aligned}$

$\Delta_{\mathrm{T}, \mathrm{h}}$ and $U_{\mathrm{S}}$ can now be determined from Eqs. (18)(24). With the aid of Eqs. (19) and (21)-(23), $\delta_{\mathrm{T}_{\mathrm{S}}, \mathrm{h}}$ can be re-expressed in terms of $U_{\mathrm{S}}$, i.e.,

$$
\delta_{\mathrm{T}_{\mathrm{S}}, \mathrm{h}} \sim \frac{\alpha}{V_{\mathrm{r}}} \sim \frac{\alpha}{A U_{\mathrm{r}}} \sim \frac{\alpha}{A \frac{\delta_{U_{\mathrm{S}}}}{D} U_{\mathrm{S}}} \sim \frac{D \alpha}{A \frac{L}{\left(\frac{U_{\mathrm{S}} L}{v}\right)^{1 / 2}} U_{\mathrm{S}}},
$$

or

$$
\delta_{\mathrm{T}_{\mathrm{S}} \mathrm{h}} \sim \frac{\alpha L^{1 / 2}}{v^{1 / 2} U_{\mathrm{S}}^{1 / 2}}
$$

By substituting Eq. (25) into Eq. (18), $\Delta_{\mathrm{T}, \mathrm{h}}$ can then be determined, which gives

$$
\Delta_{\mathrm{T}, \mathrm{h}} \sim \frac{L}{P r}
$$

From Eqs. (20) and (24), $U_{\mathrm{S}}$ can be expressed as follows:

$$
\begin{aligned}
U_{\mathrm{S}} & \sim \frac{\delta_{U_{\mathrm{S}} \mathrm{h}}\left|\hat{\sigma}_{\mathrm{T}}\right| \Delta T_{0}}{\Delta_{\mathrm{T}, \mathrm{h}} \mu} \sim \frac{\Delta_{\mathrm{T}, \mathrm{h}}}{\left(\frac{U_{\mathrm{S}} \Delta_{\mathrm{T}, \mathrm{h}}}{v}\right)^{1 / 2}} \frac{1}{\Delta_{\mathrm{T}, \mathrm{h}}} \frac{\left|\hat{\sigma}_{\mathrm{T}}\right| \Delta T_{0}}{\mu} \\
& \sim \frac{v^{1 / 2}}{U_{\mathrm{S}}^{1 / 2} \Delta_{\mathrm{T}, \mathrm{h}}^{1 / 2}} \frac{\left|\hat{\sigma}_{\mathrm{T}}\right| \Delta T_{0}}{\mu}
\end{aligned}
$$

which gives

$$
U_{\mathrm{S}}^{3 / 2} \sim \frac{v^{1 / 2}}{\Delta_{\mathrm{T}, \mathrm{h}}^{1 / 2}} \frac{\left|\hat{\sigma}_{\mathrm{T}}\right| \Delta T_{0}}{\mu}
$$

Substitution of Eq. (26) into Eq. (27) gives

$$
\begin{aligned}
U_{\mathrm{S}}^{3 / 2} & \sim \frac{v^{1 / 2}}{\frac{L^{1 / 2}}{P^{1 / 2}}} \frac{\left|\hat{\sigma}_{\mathrm{T}}\right| \Delta T_{0}}{\mu} \sim \operatorname{Pr}^{1 / 2} \frac{v^{1 / 2}}{L^{1 / 2}} \frac{\left|\hat{\sigma}_{\mathrm{T}}\right| \Delta T_{0}}{\mu} \\
& \sim \frac{\operatorname{Pr}^{1 / 2}}{A R_{\sigma, 0}^{1 / 2}} U_{0}^{3 / 2}
\end{aligned}
$$

$U_{\mathrm{S}}$ can then be derived from the above equation, i.e.,

$$
U_{\mathrm{S}} \sim \frac{\operatorname{Pr}^{1 / 3}}{A^{2 / 3} R_{\sigma, 0}^{1 / 3}} U_{0}
$$

Thus, the characteristic velocity for both the region $\Delta_{\mathrm{T}, \mathrm{h}}$ and the central portion of the cavity increases with $\operatorname{Pr}$ and decreases with $R_{\sigma, 0}$ if the aspect ratio $A$ is fixed [36,37]. However, the conventionally adopted characteristic surface velocity at the mid-point decreases with both $\mathrm{Pr}$ and $R_{\sigma, 0}$ [24]. This is because the surface temperature gradient and, thus, the thermocapillary driving force in the central portion of the cavity is very mild and the force acting on fluid particles is mainly due to viscous effect. Consequently, the surface velocity therein decreases with $\operatorname{Pr}$ and will underestimate the characteristic velocity.

With $\Delta_{\mathrm{T}, \mathrm{h}}$ and $U_{\mathrm{S}}$ determined, all the other characteristic quantities of interest in the region $\Delta_{\mathrm{T}, \mathrm{h}}$ and the central portion of the cavity can be easily derived from Eqs. (18)-(24). The corresponding surface-tension Reynolds number, $R_{\sigma, \mathrm{S}}$, and Marangoni number, $M_{a, \mathrm{~S}}$, possess the following forms:

$R_{\sigma, \mathrm{S}} \equiv \frac{U_{\mathrm{S}} L}{v}=\frac{\operatorname{Pr}^{1 / 3} R_{\sigma, 0}^{2 / 3}}{A^{2 / 3}}$

and

$M_{a, \mathrm{~S}} \equiv \operatorname{Pr} R_{\sigma, \mathrm{S}}=\frac{\operatorname{Pr}^{4 / 3} R_{\sigma, 0}^{2 / 3}}{A^{2 / 3}}=\left(\frac{\operatorname{Pr} M_{a, 0}}{A}\right)^{2 / 3}$

Before the derivation of the characteristic quantities in the small region near the cold wall is presented, one important aspect needs to be discussed first. For liquids with $\operatorname{Pr}>\mathrm{O}(1)$, the viscous diffusion is larger than the thermal diffusion. Therefore, when approaching the cold wall, the surface flow will decelerate first before the surface temperature distribution starts to descend rapidly. There thus exist two length scales even in the small region near the cold wall; one of which is for the surface flow, $\Delta_{U, \mathrm{C}}$, and the other is for the surface temperature distribution, $\Delta_{\mathrm{T}, \mathrm{C}}$. The characteristic surface velocity $U_{\mathrm{S}}$ as derived previously is appropriate for the derivation of $\Delta_{U, \mathrm{C}}$, but it is inappropriate for $\Delta_{\mathrm{T}, \mathrm{C}}$. The surface velocity used to determine $\Delta_{\mathrm{T}, \mathrm{C}}$ should be smaller than $U_{\mathrm{S}}$ according to the above discussion. Let it be denoted by $U_{\mathrm{S}}^{\prime}$. With the assumption that $A^{2} R_{\sigma, 0} \gg \mathrm{O}(1), \Delta_{U, \mathrm{C}}$ is usually very small. Using a linear variation of the surface velocity within $\Delta_{U, \mathrm{C}}$ to estimate $U_{\mathrm{S}}^{\prime}$ would be reasonable, which gives 
$U_{\mathrm{S}}^{\prime} \sim \frac{\Delta_{\mathrm{T}, \mathrm{C}}}{\Delta_{U, \mathrm{C}}} U_{\mathrm{S}}$

By balancing the inertia with the viscous effect in the $X$-direction, $\Delta_{U, \mathrm{C}}$ is determined. Then $\Delta_{\mathrm{T}, \mathrm{C}}$ can be estimated, with the aid of Eq. (32), by balancing the conduction with the convection in the $X$-direction. They are given as follows:

$\Delta_{U, \mathrm{C}} \sim \frac{v}{U_{\mathrm{S}}} \sim \frac{A^{2 / 3} R_{\sigma, 0}^{1 / 3}}{\operatorname{Pr}^{1 / 3}} \frac{v}{U_{0}} \sim \frac{A^{2 / 3}}{\operatorname{Pr}^{1 / 3} R_{\sigma, 0}^{2 / 3}} L$

and

$\Delta_{\mathrm{T}, \mathrm{C}} \sim \frac{\alpha}{U_{\mathrm{S}}^{\prime}} \sim \frac{\alpha}{\frac{\Delta_{\mathrm{T}, \mathrm{C}}}{\Delta_{U, \mathrm{C}}} U_{\mathrm{S}}}$

or

$$
\begin{aligned}
\Delta_{\mathrm{T}, \mathrm{C}} & \sim\left(\frac{\alpha \Delta_{U, \mathrm{C}}}{U_{\mathrm{S}}}\right)^{1 / 2} \sim \frac{A^{1 / 3}}{\operatorname{Pr}^{1 / 6} R_{\sigma, 0}^{1 / 3}} \frac{A^{1 / 3} R_{\sigma, 0}^{1 / 6}}{\operatorname{Pr}^{1 / 6}} \frac{\alpha^{1 / 2} L^{1 / 2}}{U_{0}^{1 / 2}} \\
& \sim \frac{A^{2 / 3}}{\operatorname{Pr}^{5 / 6} R_{\sigma, 0}^{2 / 3}} L
\end{aligned}
$$

$\Delta_{\mathrm{T}, \mathrm{C}}$ can also be expressed in terms of $M_{a, 0}$, i.e.,

$\Delta_{\mathrm{T}, \mathrm{C}} \sim \frac{A^{2 / 3}}{\operatorname{Pr}^{1 / 6} M_{a, 0}^{2 / 3}} L$

The characteristic velocity in $\Delta_{\mathrm{T}, \mathrm{C}}$ can now be determined from the tangential-force-balance boundary condition at the free surface, i.e.,

$\mu \frac{U_{\mathrm{C}}}{\delta_{U_{\mathrm{S}}}} \sim \frac{\left|\hat{\sigma}_{\mathrm{T}}\right| \Delta T_{0}}{\Delta_{\mathrm{T}, \mathrm{C}}}$

The velocity boundary layer thickness near the free surface, $\delta_{U_{\mathrm{S}}}$, due to $U_{\mathrm{S}}$, has been applied in the above equation. This is because, with $A^{2} R_{\sigma, 0} \gg \mathrm{O}(1)$, there already exists a velocity boundary layer along the free surface before the fluid enters $\Delta_{\mathrm{T}, \mathrm{C}}$. Eq. (36) then gives, with the aid of Eqs. (30) and (34),

$$
\begin{aligned}
U_{\mathrm{C}} & \sim \frac{\delta_{U_{\mathrm{S}}}}{\Delta_{\mathrm{T}, \mathrm{C}}} \frac{\left|\hat{\sigma}_{\mathrm{T}}\right| \Delta T_{0}}{\mu} \sim \frac{A^{1 / 3}}{\operatorname{Pr}^{1 / 6} R_{\sigma, 0}^{1 / 3}} \frac{\operatorname{Pr}^{5 / 6} R_{\sigma, 0}^{2 / 3}}{A^{2 / 3}} \frac{\left|\hat{\sigma}_{\mathrm{T}}\right| \Delta T_{0}}{\mu} \\
& \sim \frac{\operatorname{Pr}^{2 / 3} R_{\sigma, 0}^{1 / 3}}{A^{4 / 3}} U_{0}
\end{aligned}
$$

The pressure variation and the corresponding free surface deformation in $\Delta_{\mathrm{T}, \mathrm{C}}$ are estimated by the inertia effect and the normal-force-balance boundary condition at the free surface, respectively. They are given as follows:

$P_{\mathrm{C}} \sim \rho U_{\mathrm{C}}^{2} \sim \frac{P r^{4 / 3} R_{\sigma, 0}^{2 / 3}}{A^{8 / 3}} \rho U_{0}^{2}$

and

$$
\begin{aligned}
\eta_{\mathrm{C}} & \sim \frac{P_{\mathrm{C}}}{\hat{\sigma}_{0}} \Delta_{\mathrm{T}, \mathrm{C}}^{2} \sim \frac{\operatorname{Pr}^{4 / 3} R_{\sigma, 0}^{2 / 3}}{A^{8 / 3}} \frac{A^{4 / 3}}{\operatorname{Pr}^{5 / 3} R_{\sigma, 0}^{4 / 3}} \frac{\rho U_{0}^{2} L^{2}}{\hat{\sigma}_{0}} \\
& \sim \frac{1}{A^{4 / 3} \operatorname{Pr}^{1 / 3} R_{\sigma, 0}^{2 / 3}} A^{2} R_{\sigma, 0} \mathrm{Ca} \frac{L}{A},
\end{aligned}
$$

or

$\eta_{\mathrm{C}} \sim \frac{R_{\sigma, 0}^{1 / 3} \mathrm{Ca}}{A^{1 / 3} \operatorname{Pr} 1 / 3} L$

To determine the time scales, the following definitions are made first:

$\tau_{\mathrm{T}} \quad$ temperature time scale of the main surface flow $\tau_{\mathrm{V}} \quad$ velocity time scale of the main surface flow

$\tau_{\mathrm{T}, \mathrm{C}} \quad$ temperature time scale in $\Delta_{\mathrm{T}, \mathrm{C}}$

$\tau_{\mathrm{V}, \mathrm{C}} \quad$ velocity time scale in $\Delta_{\mathrm{T}, \mathrm{C}}$

$\tau_{\mathrm{S}} \quad$ surface variation time scale in $\Delta_{\mathrm{T}, \mathrm{C}}$

Their expressions for the present flow situation are as follows:

$$
\begin{aligned}
& \tau_{\mathrm{T}} \sim \frac{L}{U_{\mathrm{S}}} \sim \frac{A^{2 / 3} R_{\sigma, 0}^{1 / 3}}{\operatorname{Pr}^{1 / 3}} \frac{L}{U_{0}} \\
& \tau_{\mathrm{V}} \sim \frac{L}{U_{\mathrm{S}}} \sim \frac{A^{2 / 3} R_{\sigma, 0}^{1 / 3}}{\operatorname{Pr}^{1 / 3}} \frac{L}{U_{0}} \\
& \tau_{\mathrm{T}, \mathrm{C}} \sim \frac{\Delta_{\mathrm{T}, \mathrm{C}}}{U_{\mathrm{C}}} \sim \frac{A^{2 / 3}}{\operatorname{Pr}^{5 / 6} R_{\sigma, 0}^{2 / 3}} \frac{A^{4 / 3}}{\operatorname{Pr}^{2 / 3} R_{\sigma, 0}^{1 / 3}} \frac{L}{U_{0}} \sim \frac{A^{2}}{\operatorname{Pr}^{3 / 2} R_{\sigma, 0}} \frac{L}{U_{0}} \\
& \sim \frac{A^{2}}{\operatorname{Pr}^{1 / 2} M_{a, 0}} \frac{L}{U_{0}} \\
& \tau_{\mathrm{V}, \mathrm{C}} \sim \frac{\Delta_{\mathrm{T}, \mathrm{C}}}{U_{\mathrm{C}}} \sim \frac{A^{2}}{P^{3 / 2} R_{\sigma, 0}} \frac{L}{U_{0}} \sim \frac{A^{2}}{\operatorname{Pr}^{1 / 2} M_{a, 0}} \frac{L}{U_{0}} \\
& \tau_{\mathrm{S}} \sim \frac{\eta_{\mathrm{C}} \Delta_{\mathrm{T}, \mathrm{C}}}{U_{\mathrm{S}} \delta_{U_{\mathrm{S}}}} \\
& \sim \frac{R_{\sigma, 0}^{1 / 3} \mathrm{Ca}}{A^{1 / 3} \operatorname{Pr}^{1 / 3}} \frac{A^{2 / 3}}{\operatorname{Pr}^{5 / 6} R_{\sigma, 0}^{2 / 3}} \frac{A^{2 / 3} R_{\sigma, 0}^{1 / 3}}{\operatorname{Pr}^{1 / 3}} \frac{\operatorname{Pr}^{1 / 6} R_{\sigma, 0}^{1 / 3}}{A^{1 / 3}} \frac{L}{U_{0}} \\
& \sim \frac{A^{2 / 3} R_{\sigma, 0}^{1 / 3} C a}{\operatorname{Pr}^{4 / 3}} \frac{L}{U_{0}}
\end{aligned}
$$

\section{Concluding remarks}

An unsteady, two-dimensional thermocapillary convection with a deformable free surface in a rectangular cavity is analyzed. Four flow regimes defined in the present study are: (A) situations with $A^{2} M_{a, 0} \leqslant \mathrm{O}(1)$ and $A^{2} R_{\sigma, 0} \leqslant \mathrm{O}(1)$, (B) situations with $A^{2} M_{a, 0} \leqslant \mathrm{O}(1)$ and $A^{2} R_{\sigma, 0} \gg \mathrm{O}(1)$, (C) situations with $A^{2} M_{a, 0} \gg \mathrm{O}(1)$ and $A^{2} R_{\sigma, 0} \leqslant \mathrm{O}(1)$, and (D) situations with $A^{2} M_{a, 0} \gg \mathrm{O}(1)$ and $A^{2} R_{\sigma, o} \gg \mathrm{O}(1)$. The temperature distribution and flow structure are different from one flow regime to another. The multiple-scale analysis based on appro- 
priate physical arguments has been applied to determine the characteristic length, time, and velocity scales and obtain a global picture for each flow regime by Lai [35]. However, only the analysis for the flow situations with $A^{2} M_{a, 0} \gg \mathrm{O}(1), A^{2} R_{\sigma, 0} \gg \mathrm{O}(1)$, and $\operatorname{Pr}>\mathrm{O}(1)$ is presented herein because it is the flow regime where the experimental data for the onset of oscillatory thermocapillary convection are available. One very important and interesting result of the present study is concluded in the following.

The ratio of the surface-variation time scale to the convective time scale of the main surface flow can be obtained from Eqs. (40) and (44), which gives the following relation:

$\frac{\tau_{\mathrm{S}}}{\tau_{\mathrm{T}}} \sim \frac{C a}{P r}$

Since $\tau_{\mathrm{T}}=\tau_{\mathrm{V}}$, this relation also implies

$\frac{\tau_{\mathrm{S}}}{\tau_{\mathrm{V}}} \sim \frac{C a}{P r}$

The right-hand sides of Eqs. (45) and (46) is the "surface-deformation number" or the so-called " $S$-number" claimed by Ostrach and Kamotani [12,31] and first derived by Lai [30] based on several physical conjectures. The validity of $S$-number has been justified by good and consistent correlation among the on-ground and outerspace experimental data, and has been presented and published previously [12,30-32].

As proposed and verified by Lai [19,30], the surface deformation will result in a time lag of the return flow which provides a cooling effect on the surface flow. Eqs. (45) and (46), or, the $S$-parameter, therefore indicates, in a sense, a delayed cooling effect of the return flow on the surface temperature distribution of the main flow. This delayed cooling effect of the return flow might result in an overshooting and undershooting of the surface temperature distribution near the hot wall (vs. the steady equilibrium state) and, hence, induce an oscillatory thermocapillary convection as observed in the experiments.

Most important, the present study provides a theoretical basis for the derivation of the $S$-parameter. Because most the experimental data, either terrestrial or outer-space, are all well correlated by the $S$-parameter, it is therefore believed that the surface deformation is crucial to the onset of oscillatory thermocapillary convection. It is recommended herein that more effort and attention need to be directed toward the study of oscillatory thermocapillary convection with a deformable free surface.

\section{Acknowledgements}

Support from the National Center for Microgravity Research on Fluids and Combustion is greatly acknowledged.

\section{References}

[1] S. Ostrach, Low-gravity fluid flows, Annu. Rev. Fluid Mech. 14 (1982) 313-345.

[2] N.O. Young, J.S. Goldstein, M.J. Block, The motion of bubbles in a vertical temperature gradient, J. Fluid Mech. 6 (1959) 350-356.

[3] J.M. Papazian, R. Gutowski, W.R. Wilcox, Bubble behavior during solidification in low gravity, AIAA J. 17 (1979) 1111-1117.

[4] R.S. Subramanian, Slow migration of a gas bubble in a thermal gradient, AIChE J. 7 (1981) 646-654.

[5] W.A. Sirignano, L. Glassman, Flame spreading above liquid fuels: surface tension flows, Combust. Sci. Technol. 1 (1970) 307-312.

[6] G.M. Oreper, T.W. Eagar, J. Szekely, Convection in weld pools, Welding J. 62 (1983) 307-312.

[7] D. Schwabe, Marangoni effects in crystal growth melts, Physicochem. Hydrodynam. 2 (1981) 263-280.

[8] R. Balasubramanian, S. Ostrach, Fluid motions in the Czechruski method of crystal growth, Physicochem. Hydrodynam. 5 (1984) 3-18.

[9] S. Ostrach, Motion induced by capillarity, Physicochem. Hydrodynam. 2 (1977) 571-589.

[10] Ch.H. Chun, Experiments on steady and oscillatory temperature distribution in a floating zone due to the Marangoni convection, Acta Astronaut. 7 (1980) 479-488.

[11] D. Schwabe, A. Scharmann, R. Preisser, Verification of the oscillatory state of thermocapillary convection in a floating zone under low gravity, Acta Astronaut. 9 (1981) 265-273.

[12] Y. Kamotani, S. Ostrach, M. Vargas, Oscillatory thermocapillary convection in a simulated floating-zone configuration, J. Cryst. Growth 66 (1984) 83-90.

[13] A.G. Kirdyashkin, Thermocapillary periodic flows, Int. J. Heat Mass Transfer 30 (1) (1987) 109-124.

[14] J. Masud, Y. Kamotani, S. Ostrach, Oscillatory thermocapillary flow in cylindrical columns of high Prandtl number fluids, J. Thermophys. Heat Transfer 11 (1) (1997) 105-111.

[15] S.M. Pimputkar, S. Ostrach, Transient thermocapillary flow in thin layers, Phys. Fluids 23 (1980) 1281-1285.

[16] A.K. Sen, S.H. Davis, Steady thermocapillary flows in twodimensional slots, J. Fluid Mech. 121 (1982) 163-186.

[17] S. Ostrach, Y. Kamotani, C.L. Lai, Oscillatory thermocapillary flows, Physicochem. Hydrodynam. 6 (1985) 585-599.

[18] A.K. Sen, Thermocapillary convection in a rectangular cavity with a deformable interface, Phys. Fluids 29 (1986) 3851-3853.

[19] C.L. Lai, Unsteady thermocapillary flows and free surface oscillations in reduced gravity environments, Acta Atronaut. 21 (3) (1990) 171-181.

[20] C.L. Lai, T.S. Yang, Unsteady thermocapillary flows and free surface oscillations, Acta Astronaut. 21 (9) (1990) 629640.

[21] B.I. Fu, S. Ostrach, Numerical solutions of floating-zone thermocapillary flows, in: Proceedings of the 4th European Symposium on Materials Sciences Under Microgravity, Madrid, Spain, 1983, pp. 239-245.

[22] N. Kobayashi, Computer simulation of the steady flow in a cylindrical floating zone under low gravity, J. Cryst. Growth 66 (1984) 63-72. 
[23] M.K. Smith, The nonlinear stability of dynamic thermocapillary liquid layers, J. Fluid Mech. 194 (1988) 391415.

[24] B.M. Carpenter, G.M. Homsy, High Marangoni number convection in a square cavity: Part II, Phys. Fluids 2 (1990) 137-149.

[25] M. Levenstam, G. Amberg, Hydrodynamical instabilities of thermocapollary flow in a half-zone, J. Fluid Mech. 297 (1995) 357-372.

[26] M. Wanschura, V.M. Shevtsova, H.C. Kuhlmann, H.J. Rath, Convective instability mechanisms in thermocapillary liquid bridges, Phys. Fluids 7 (1995) 912-925.

[27] V.M. Ha, C.L. Lai, Study of Marangoni instability of an evaporating liquid layer, J. Chinese Inst. Engrs. 21 (5) (1998) 547-562.

[28] T.P. Hsieh, Thermocapillary instability of a deformable liquid layer subjected to transverse disturbances, M.S. Thesis, Department of Mechanical Engineering, National Taiwan University, Taiwan, ROC, 1999.

[29] V.M. Ha, C.L. Lai, The onset of stationary Marangoni instability of an evaporating droplet, Proc. R. Soc. Lond. A 457 (2001) 885-909.

[30] C.L. Lai, Studies of thermocapillary oscillation phenomena, Ph.D. Dissertation, Department of Mechanical And Aerospace Engineering, Case Western Reserve University, 1984.
[31] Y. Kamotani, S. Ostrach, Theoretical analysis of thermocapillary flow in cylindrical columns of high Prandtl number fluids, ASME J. Heat Transfer 120 (3) (1998) $758-764$.

[32] Y. Kamotani, S. Ostrach, J. Masud, Microgravity experiments and analysis of oscillatory thermocapillary flows in cylindrical containers, J. Fluid Mech. 410 (2000) 211-233.

[33] S. Ostrach, Convection due to surface-tension gradients, in: M.J. Rycroft (Ed.), COSPAR Space Research, vol. 19, 1979 , pp. $563-570$.

[34] D.D. Hu, Thermocapillary flows in an enclosure of unit order aspect ratio, Ph.D. Dissertation, Department of Mechanical And Aerospace Engineering, Case Western Reserve University, 1990.

[35] C.L. Lai, Multiple-scale analysis on oscillatory thermocapillary flows, The National Center for Microgravity Research on Fluids and Combustion, Case Western Reserve University, 1998.

[36] Being-I Fu, Numerical solutions of floating zone crystal growth, Ph.D. Dissertation, Department of Mechanical and Aerospace Engineering, Case Western Reserve University, Cleveland, OH, 1983.

[37] K.C. Chen, Thermo-diffusocapillary flows in a two-dimensional rectangular cavity, Master thesis, Department of Mechanical Engineering, National Taiwan University, Taiwan, ROC, 1988. 